http://revistas.uchile.cl/index.php/RU/index

\title{
Explorando el hacinamiento en condominios sociales.
}

Exploring overcrowding in social housing condominiums.

Marco Fuentes Maturana

\section{Filiación}

Facultad de Arquitectura y Urbanismo, Universidad de Chile.

\begin{abstract}
Resumen
Los condominios sociales ${ }^{1}$, conocidos genéricamente como "block" de departamentos, han ido evidenciando con el paso de los años una serie de deficiencias. Estas se han materializado en ampliaciones irregulares, apropiaciones de terrenos, fallas constructivas, entre varias otras. El presente artículo es parte de una investigación realizada el año 2011, que buscó indagar en los problemas que presenta cierta tipología de condominio social, para posteriormente aportar en la búsqueda de soluciones frente a tales inconvenientes. A continuación, se exponen las principales observaciones rescatadas de dicho proceso, el cual se realizó a través de entrevistas semi estructuradas a habitantes de dos conjuntos sociales de la región metropolitana. Finalmente, y de modo complementario a las conclusiones se reflexiona respecto a ciertas normativas que deberían ser revisadas con el fin de evitar que se repliquen los problemas detectados en los dos casos de estudio.
\end{abstract}

\section{Palabras Claves}

Hacinamiento, condominios sociales, habitabilidad.

\begin{abstract}
Through time, social housing condominiums, commonly known as "apartment blocks", have evidenced a series of deficiencies that have become tangible through the construction of irregular renovations, the illegal appropriation of sites, the poor quality of the built form, etc. This article is part of a research project, done in 2011, that looked into the problems that the afore mentioned social housing typology presents, in order to propose solutions to them.

This article is a synthesis of the fundamental observations retrieved in the research process, done through a series of semi-structured interviews to the inhabitants of two social housing complexes in the Metropolitan Region of Santiago, which concludes with a series of recommendations regarding the revision of the urban regulations that are currently in place.
\end{abstract}

\section{Key Words}

Overcrowding, social housing condominium, habitability

\author{
Sumario \\ Introducción \\ Objetivos \\ Algunas consideraciones: Hacinamiento y habitabilidad \\ Metodología y casos de estudio \\ Principales resultados
}

\footnotetext{
${ }^{1}$ Aquellas viviendas sociales que forman parte de una copropiedad, es decir, que junto a los bienes exclusivos, existen bienes de dominio común pertenecientes a todos los copropietarios los cuales resultan necesarios para la existencia, seguridad y conservación del condominio o edificio en cuestión (MINVU, 2012). 
ISSN 0717-5051

http://revistas.uchile.cl/index.php/RU/index

\section{Conclusiones}

Recomendaciones

Bibliografía

\section{INTRODUCCIÓN}

En las últimas dos décadas, la política de vivienda social de Chile permitió disminuir de forma considerable el déficit habitacional. En el periodo 1996-2007 el número de familias habitando en campamentos disminuyó de 59.053 a 20.509. Posteriormente hacia el 2011 tal cifra se elevó levemente a las 27.378 familias (MINVU, 2011). Por otro lado, respecto a las viviendas construidas, es que las fuentes oficiales informaron el 2004 la existencia de 543.542 "requerimientos", refiriéndose de esta forma "a las viviendas que falta construir en el país para reponer las viviendas irrecuperables, de modo de entregar alojamiento a los hogares allegados y dotar de vivienda a las familias secundarias que enfrentan problemas urgentes de calidad de vida, y que están en condiciones de independizarse" (MINVU, 2004: 89). Es decir, entre el periodo 1992-2002 la necesidad de construcción de nuevas viviendas disminuyó de 771.935 a 543.452 (MINVU, 2004 (2)).

Sin embargo, y a pesar de que los datos censales con los que contamos indican que el déficit habitacional ha disminuido considerablemente, también es claro que las soluciones habitacionales entregadas en las últimas décadas presentan serios problemas necesarios de abordar. Muchos de estos conjuntos se caracterizan por una baja calidad en su infraestructura, y por una gran cantidad de apropiaciones irregulares que terminan por aniquilar el espacio público. El estudio elaborado por la Corporación de Estudios Sociales y Educación, muestra que en Chile, el $40 \%$ de los usuarios de vivienda social realizan algún tipo de ampliación a sus moradas, en donde "la gran mayoría de los beneficiarios con techo construyen algo adicional, casi tan grande como la vivienda original" (Rodríguez y Sugranyes, 2005: 65).Tal situación evidencia cierta carencia espacial que se ve incrementada por las características del entorno inmediato. En general, estos espacios están en constante deterioro (Aravena y Sandoval, 2005) presentando un déficit en equipamientos e ineficientes diseños. A lo anterior debe sumarse que muchos de estos conjuntos, producto de la subordinación de la política de la vivienda al mercado de suelo urbano, han sido objetos de una aguda segregación socioeconómica residencial (Trivelli, 2009), en donde las "nuevas villas" se han ido localizando en lugares "marginales" como una expresión más de segregación residencial" (Aravena y Sandoval, 2005). Tales observaciones pueden ser corroborado fácilmente visitando los condominios sociales repartidos por la región metropolitana, en donde muy probablemente se observara ropa colgada en las escaleras de acceso, cierres y rejas resguardando tanto las viviendas como los ingresos a los bloques y una gran cantidad de ampliaciones y apropiaciones. Tales situaciones, se observan generalmente en zonas con bajo equipamiento, sin espacios públicos consolidados y muchas veces alejados de los centros comerciales y de servicios.

Lo anteriormente expuesto se materializa como el motivo gestor de la investigación que da origen al presente artículo. Dicha investigación se inscribe como una exploración dentro de las posturas que plantean que es necesario cambiar el foco en las políticas públicas, comenzando a centrar la mirada no solo en terminar con el déficit habitacional (entendido habitualmente como la necesidad de proveer viviendas), sino también en mejorar el parque habitacional que se encuentra altamente deteriorado en Chile. Se comparten las posturas de Rodríguez y Sugranyes (2005), quienes han afirmado que "hoy en Santiago, el gran problema social 
habitacional es el de las familias "con techo"" (pág. 60), haciendo referencia a aquellos grupos que habitan las viviendas sociales de nuestro país. Tal grupo, según los autores recién mencionados no es menor, sumando según sus estudios a alrededor de 1 millón de personas.

Para mejorar este parque habitacional, resulta necesario comprender el problema y entender la percepción que tienen los habitantes respecto a sus propios conjuntos. Con dicho propósito, es que el estudio mencionado se dividió en dos etapas, la primera buscó estudiar el fenómeno del hacinamiento y la reducida superficie de los condominios sociales de nuestro país, mientras que la segunda se centró en la exploración de soluciones para tal problemática.

Se decidió estudiar este fenómeno en los condominios sociales, dado a que dentro del parque habitacional de vivienda social, dicha tipología destaca por presentar una serie de dificultades dada sus características administrativas, legales y funcionales. Este problema se vuelve más evidente, cuando observamos que en la actualidad no hay experiencias de ampliaciones de condominios sociales llevadas a cabo por el Estado. Existe un par de casos en la región metropolitana, destacando la realizada en el conjunto Los Quillayes de la comuna de La Florida, en donde se lograron ampliar 15 departamentos gracias al trabajo en conjunto del INVI, una ONG, la respectiva Municipalidad, el estado a través de los subsidios entregados, entre otros (INVI, 2005). Dentro de la gama de tipologías que presenta dicha solución habitacional se ha escogido aquella caracterizada por estar formada por bloques paralelos con escaleras de "tijeras" en su interior, dado a que es una de las más repetidas en la región metropolitana. Además, esta tipología se ha caracterizado por una serie de problemáticas tanto sociales como en relación a su configuración espacial, dado entre otros aspectos por "la máxima racionalización de recursos con que fueron desarrollados dichos proyectos habitacionales" (INVI, 2005: 41).

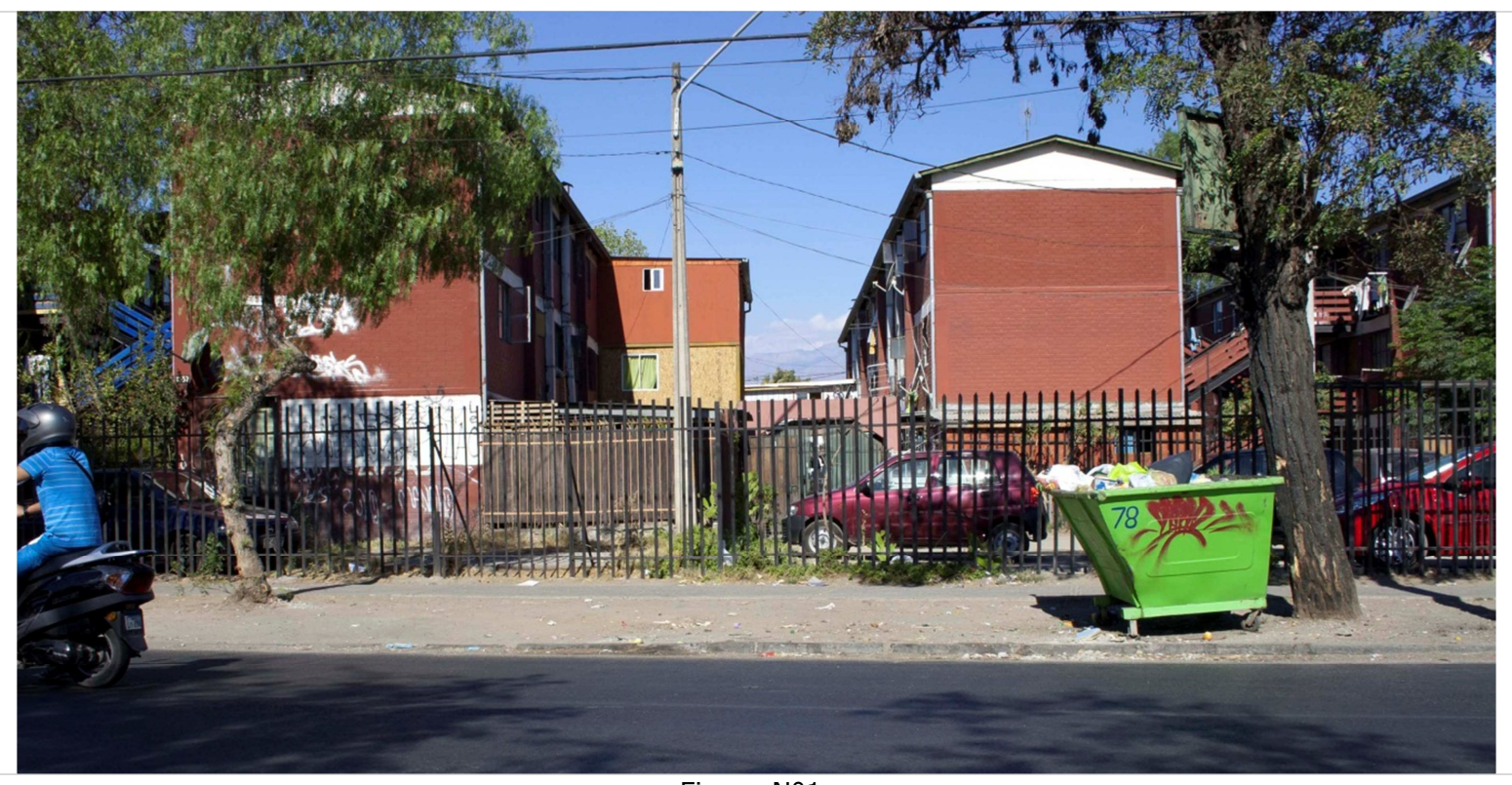

Figura No1:

Conjunto San Alberto Hurtado, Lo Prado: Ejemplo de la tipología seleccionada Fuente: Elaboración Propia. 


\section{OBJETIVOS}

El estudio citado, planteó como objetivo general explorar la problemática del hacinamiento que presentan los condominios sociales en Chile, de manera de entender sus causalidades arquitectónicas y urbanas $y$, de esta forma, entregar recomendaciones que puedan ser traducidas en posibles soluciones de diseño y planificación urbana estratégica.

Se plantea como hipótesis, (1) que las características internas de los condominios sociales no logran satisfacer plenamente las condiciones de habitabilidad, encontrándose muchos de estos conjuntos en situación de hacinamiento. (2) Este hipotético hacinamiento no solo afecta la vida al interior de la vivienda sino que repercute negativamente en el espacio exterior, siendo no solo consecuencia de un hábitat residencial deficiente, sino también causa, ya que en la medida que una familia trata de salir de dicha situación repercute negativamente en la habitabilidad de los conjuntos. Se plantea también a modo de hipótesis (3) que las viviendas en situación de hacinamiento son muchas más que lo que indican las fuentes oficiales, las cuales hablan de alrededor de un $17,16 \%$ del total de viviendas del país.

Objetivos específicos:

(1) Explorar si las condiciones de habitabilidad que el hacinamiento vulnera están siendo transgredidas, según la percepción de los habitantes de Condominios Sociales.

(2) Observar el impacto a nivel urbano que produce el hacinamiento en condominios sociales.

(3) Analizar en qué medida la percepción de hacinamiento y por lo tanto de un hábitat residencial defectuosos, se intensifica o aminora de acuerdo a la localización del conjunto dentro del Gran Santiago.

(4) Aportar en la búsqueda de soluciones para las familias que se encuentran en situación de hacinamiento.

\section{ALGUNAS CONSIDERACIONES: HACINAMIENTO Y HABITABILIDAD}

En la elaboración del marco teórico que sustentó la investigación se desarrollaron ciertos temas importantes de mencionar. En primer lugar, se cuestionó el sistema de medición del hacinamiento utilizado en Chile, ya que este se realiza en torno a la cantidad de personas habitando en un dormitorio, independiente de la calidad o superficie de la vivienda ${ }^{2}$. Se considera necesario desprenderse de esta definición numérica tratando de entender y caracterizar las consecuencias que el hacinamiento provoca. Con este objetivo es que se revisó una serie de autores que se han referido a esta patología con el objeto de caracterizar tal problemática. Es así como el hacinamiento se entenderá como una patología que producto de una "densidad que sobrepasa ciertos límites tolerables" (Puga, 1983: 202) puede provocar alteraciones tanto a nivel de individuo, familiar o de conjunto habitacional. Como se explica a continuación tales alteraciones tienen que ver fundamentalmente con la privacidad y la territorialidad que los diferentes grupos deberían lograr establecer.

Frente a la insuficiencia de espacio las personas pueden verse "obligadas a comportamientos, relaciones o descargas emocionales en extremo estresantes" (Lentini y Palero, 1997: 26). Tal

${ }^{2}$ una vivienda presenta hacinamiento medio si existe entre 2,5 y 4,9 personas por dormitorio, y presentan hacinamiento crítico si se encuentran más de 5 personas por dormitorio (MINVU, 2004) 
problemática es una de las principales causas de la vida en "la calle" en donde niños y adolescentes se ven expuestos a ciertos peligros y conductas no adecuadas para su formación (Martínez, 2001). Por otro lado, y a nivel de individuo, se pueden producir alteraciones en la salud física, al facilitarse la transmisión de enfermedades infecto-contagiosas, y en lo psíquico, al provocarse "alteraciones del carácter, como tensiones, irritaciones y desequilibrios nerviosos" (Puga, 1983: 205).

En la escala familiar, se observan dificultades de carácter doméstico tales como el guardado de objetos, o la falta de espacio para la realización de actividades cotidianas como leer, estudiar o escuchar música. Genera dificultades en las dinámicas internas, como problemas para que los niños jueguen, para la intimidad de la pareja, entre otras (Martínez, 2001). En el caso de las relaciones intrafamiliares, se obstaculiza "el reconocimiento que cada miembro espera del status que ocupa en el grupo, según sea su rol, su sexo y su edad" (Puga, 1983).

Es posible afirmar que las anteriores dificultades se relacionan directamente con la privacidad que los individuos necesitan. Autores como Martínez (2001) o Harrison (1993) destacan justamente el impacto que genera el hacinamiento en tal condición. Este último autor menciona la necesidad de carácter individual que tienen los seres humanos de controlar las interacciones con los demás para el encuentro y conocimiento de uno mismo. Puga (1983) afirma que la privacidad es un bien necesario para la persona, "quien necesita a menudo abstraerse de sus roles familiares para ser ella misma" (pág. 205).

Esta privacidad tan necesaria para las personas, no es puramente individual, la familia necesita diversos grados de privacidad en relación con personas ajenas a su núcleo. La forma de recibir a un pariente es distinta a la forma de recibir a un semidesconocido, y "el uso de los recintos se les permitirá en distintas formas a uno y a otro" (Harrison, 1993: 163). Rugiero (2000) comenta que esta necesidad de establecer cierta separación con el exterior ha sucedido históricamente, indicando que es normal observar como las viviendas urbanas incorporaban un muro en el acceso de tal forma de asegurar la privacidad visual.

Por otra parte, el hacinamiento así como altera la privacidad de las familias, evitando el correcto desenvolvimiento de funciones tanto personales como grupales, interfiere en la "territorialidad" que los usuarios establecen del espacio habitado (Lentini y Palero, 1997). Entendiéndose territorialidad, como la "toma de posesión, utilización y defensa de un territorio por parte de los organismos vivos" (Hall, 1989: 58).

Como se observa, y a modo de síntesis, para esta investigación se decidió entender el hacinamiento sin considerar la relación cantidad de personas $\mathrm{v} / \mathrm{s}$ dormitorio, sino que desde el punto de vista de las consecuencias que esta problemática ocasiona. De esta forma, se buscó ver en qué medida la falta de espacio afecta en la habitabilidad de los usuarios. "Habitabilidad" que también se desprendió de aquella definición tradicional que la relaciona con un estándar, normas o reglas para asegurar ciertas condiciones básicas en materia de vivienda. Se optó por una definición planteada por un grupo de autores españoles (Arcas et. al. 2011) quienes la definen desde la "sostenibilidad". Tales investigadores proponen que para lograr habitabilidad, los procesos deben estar subordinados a la limitación de recursos y de los residuos generados, siendo necesario enunciar dicho concepto desde las personas y sus necesidades. Además consideran necesario tomar conciencia respecto a la existencia de diversos modos de vida o estructuras familiares. Finalmente, al referirse a la habitabilidad proponen que esta debe plantearse fuera de los límites de la vivienda y del entorno inmediato para alcanzar una escala urbana mayor (Arcas et. al. 2011). Tal propuesta recuerda los planteamientos del INVI, 
http://revistas.uchile.cl/index.php/RU/index

quienes al referirse a la vivienda prefieren hablar de un hábitat residencial compuesto por 3 dimensiones o escalas territoriales: el conjunto, el entorno inmediato y la vivienda propiamente tal (INVI, 2004).

\section{METODOLOGÍA Y CASOS DE ESTUDIO}

La investigación presentó un sustento metodológico de tipo cualitativo, orientado en la obtención de una imagen real de la habitabilidad de los condominios sociales, focalizado esencialmente en el aparente hacinamiento que estos presentan. Para tal misión se realizaron entrevistas semi-estructuradas a los residentes de dos conjuntos de la región metropolitana. Como se mencionó en la introducción, la tipología de condominio social que se decidió estudiar fue aquella caracterizada por estar formada por dos bloques ubicados en forma paralela con escaleras "tipo tijeras" entre ellos. Esto dado a que es una de la tipología más repetida en la región metropolitana (Gutierez, 2010) en donde la disposición espacial de los bloques genera importantes problemas espaciales (Poblete, 2001). Dentro de las posibilidades de agrupamiento de dichos pares de bloques se escogió aquella conocida como disposición en "peineta", caracterizada por conformarse por una sucesión de pares de bloques (ver figura 2) en que los problemas espaciales se intensifican dado a la aparición de mayores espacios residuales inutilizados.

Los entrevistados fueron seleccionados de acuerdo a la situación del departamento en el conjunto habitacional. Es decir, se procuró extraer testimonios de los integrantes de familias habitantes de las 4 tipologías de viviendas consideradas necesarias para la investigación: departamento ubicado en primeros pisos (ampliados y no ampliados) y departamentos en pisos superiores (ampliados y no ampliados).

Se escogieron dos conjuntos con similares características en materia de cantidad de departamentos (alrededor de $180 \mathrm{c} / \mathrm{u}$ ) y de bloques (alrededor de $10(\mathrm{c} / \mathrm{u}$ ), incorporando la variable localización dentro de la región metropolitana. De esta forma los criterios de elección que diferencian los dos conjuntos fueron básicamente la ubicación respecto al anillo Américo Vespucio, la conectividad hacia el centro de Santiago, la distancia hacia sub-centros de servicios y el equipamiento de las respectivas zonas en que se ubican. De esta forma, el primer condominio escogido corresponde al conjunto Alberto Hurtado de la comuna de Lo Prado, caracterizado por su ubicación central dentro del Gran Santiago, cercano a la estación de metro Lo Prado (alrededor de 600 metros), dentro del anillo Américo Vespucio, y cercano a una serie de servicios y equipamientos comunitarios. El conjunto limita con la avenida Neptuno y la calle Lago Lanalhue en el sentido norte- sur, y con las calles Lago Ranco y Nueve de Julio en el sentido oriente -poniente.

Por el contrario, el segundo conjunto elegido fue Millaray II, de la comuna de La Pintana, ubicado fuera del anillo Américo Vespucio (a $2.1 \mathrm{~km}$ de este en su distancia más corta), en una zona caracterizada por un deficiente equipamiento comunitario, encontrándose alejado de importantes servicios. El conjunto, el cual limita con zonas no urbanizadas (de carácter agrícola), posee una superficie de 1 hectárea limitando en el sentido norte-sur con las calles Las Parcelas y Los Olmecas y en sentido oriente-poniente con la calle Machitún y la avenida General Arriegada.

Del total de familias (de ambos conjuntos) se entrevistó a poco más del $10 \%$ de los departamentos (al menos 1 integrante por vivienda), realizándose finalmente 44 entrevistas. 


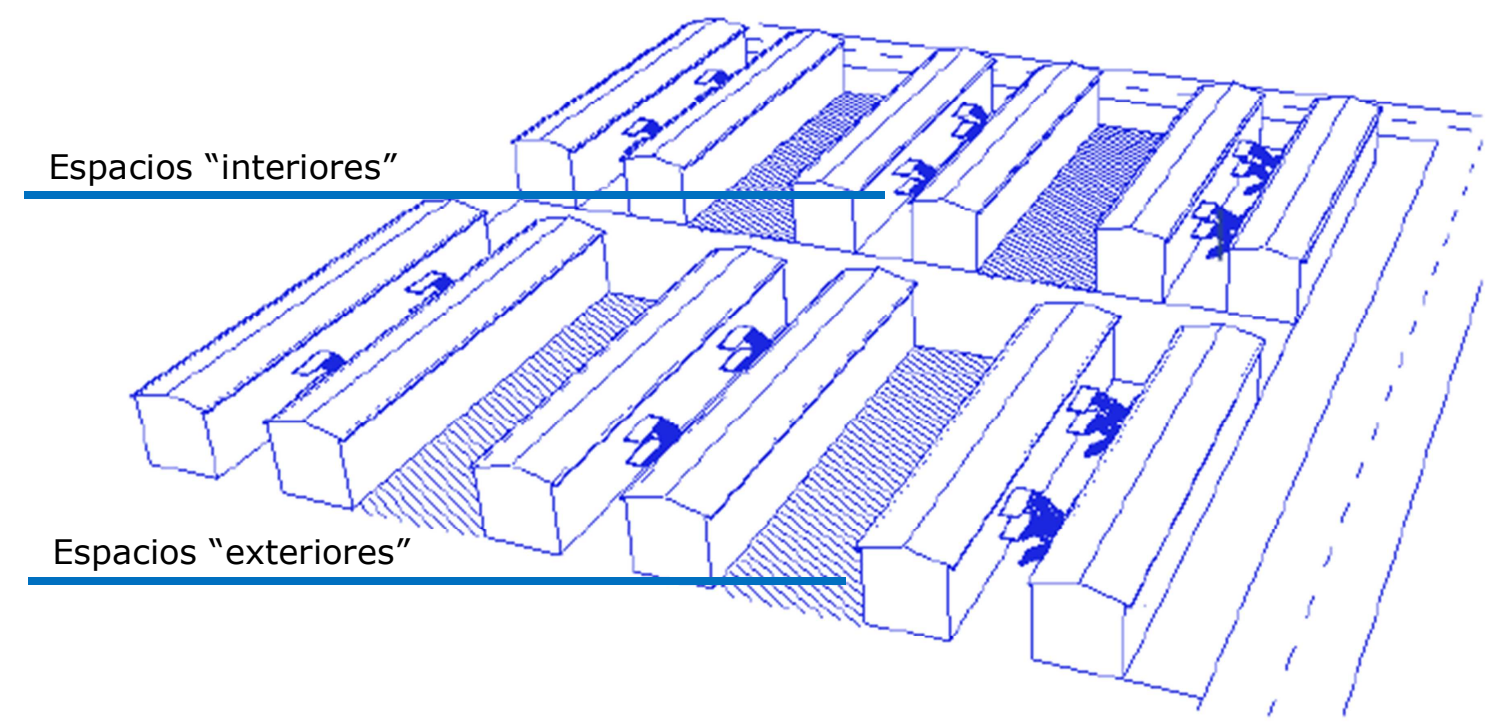

Figura No2:

Esquema conjunto agrupación en peineta.

Fuente: Elaboración Propia.

\section{PRINCIPALES RESULTADOS}

Considerando lo planteado anteriormente respecto al hacinamiento y la habitabilidad, es que se expondrán los resultados obtenidos de las entrevistas a los vecinos de los 2 conjuntos estudiados. Los siguientes son observaciones que buscan caracterizar los principales problemas en relación al hacinamiento.

1. En primer lugar y de forma transversal a los dos conjuntos, se observa una serie de problemas entre los vecinos originados principalmente por el sistema en copropiedad en que están inmersos. Se observa cierto consenso respecto al deseo de vivir en una casa y no en departamento. Más del $80 \%$ de los entrevistados contestó que si pudieran, de inmediato se cambiarían a una casa. En general, se podría plantear que la causa de esto tiene que ver con una serie de conductas que hace que los vecinos se sientan invadidos tanto en el interior como en el exterior de sus viviendas. Los entrevistados argumentaron, entre otros, problemas en relación a la vulneración de la tranquilidad causada por el ladrido de perros, el volumen alto de música, la realización de asados y fiestas y discusiones entre vecinos o a nivel intrafamiliar. Además de esto, debe sumarse una constante molestia por la falta de conciencia respecto a los deberes de los individuos. En general se observa poca responsabilidad respecto a los espacios de dominio común. Así lo afirmaron una serie de residentes al comentar que sus vecinos no se hacían cargo de la limpieza de los espacios, de los desordenes generados por sus hijos, de 
respetar las zonas de estacionamientos o por la mantención de bienes comunes como rejas, puertas o pavimentos.

En el caso de Lo Prado, se observa gran cantidad de discusiones por el uso que los residentes hacen del espacio comunitario (aquel donde se ubican las escaleras de acceso). Se observa un exceso de demanda para la superficie que poseen los "espacios interiores". En dicho lugar se tratan de realizar las actividades que las viviendas no permiten, principalmente por el tamaño de estas y por la falta de recintos como logias o garaje. Los vecinos ven tal zona como propicia para colgar ropa (principalmente en las escaleras), para estacionar los vehículos, para que sus hijos jueguen o para poder descansar. Como se observa, todas estas actividades son incompatibles una de otras, siendo cada una de ellas válidas y necesarias para dichas familias. La falta de organización, la inexistencia de juntas de vecinos o administraciones y la mala comunicación entre los vecinos, contribuyen en el desorden del conjunto. Lo anteriormente mencionado ha motivado el deseo de una vivienda independiente en que cada familia pueda desarrollar todas las actividades recién mencionadas sin interferir en la tranquilidad de sus pares.

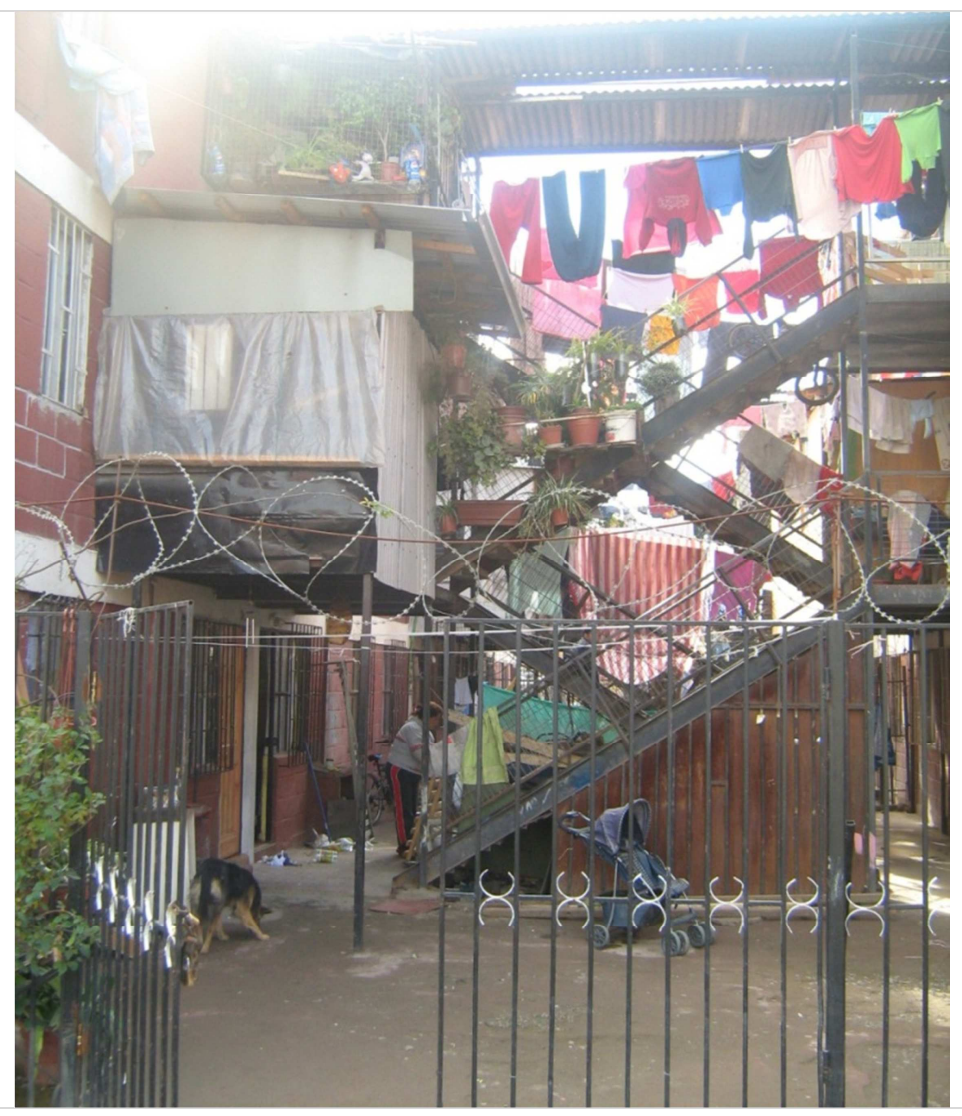

Figura $\mathrm{N}^{\circ} 3$ :

Conjunto Caupolicán San Valentín Fuente: Elaboración Propia.

2. Respecto a aquellas tendencias comunes en ambos conjuntos, es que se observa, al igual que en muchos otros condominios sociales de la región, como prácticamente el $100 \%$ de los 
usuarios del primer nivel ha ampliado sus viviendas. Los espacios traseros (aquellos donde no se ubican las escaleras de acceso) se caracterizan por ser zonas sin actividad ni control, lo que ha generado una progresiva apropiación por parte de los residentes de los primeros niveles. Esta situación evidencia la falta de conciencia respecto a que este territorio es de uso común y por tanto debería ser utilizado como tal. Los entrevistados entienden como normal tal fenómeno ignorando o despreciando su cualidad de espacio público. Lo anterior sucede a pesar de los conflictos y de la gran cantidad de necesidades que los residentes deben satisfacer en el medio exterior. Si bien dichas zonas poseen la superficie para poder paliar tales deficiencias, la configuración monótona de tales espacios, ubicados y proporcionados de manera ineficiente, producen que tales lugares no sean utilizados, ni mucho menos valorados.

3. Lo anterior, además de dar cuenta de un espacio exterior ineficiente y poco valorado, permite suponer que hay problemas de espacio importante al interior de las viviendas. Tal afirmación logra sustentarse luego de que los diferentes entrevistados comentaran las complejidades en el diario vivir que se generan producto de la superficie de los departamentos.

"nosotros ahora somos 8 personas, mis hijos están de allegados, imagínese como es cuando estamos todos, yo tengo 4 piezas, pero son todas chicas, las camas están plegadas a la pared, hay que ingeniárselas para hacer el aseo, tengo que levantar la cama para poder barrer, a mi me gustaría ampliarme, no para mis hijos, porque tengo el sueño de que ellos tengan su casa propia, sino para mí, para tener mi metro cuadrado, mi espacio". (Cecilia Sepúlveda, habitante de Lo Prado)

Los entrevistados comentaban que si bien tienen un techo donde cobijarse (lo cual en general es motivo de orgullo), este no les permite la realización de simples actividades, ni cuentan con la privacidad necesaria para poder estar solos, tranquilos o como expuso una de las entrevista, donde pudiesen "estar con ellos mismos". Prácticamente el $100 \%$ de los entrevistados en algún momento de la conversación se refirió al tamaño de las viviendas, lo cual de alguna u otra forma significa o había significado en el transcurso de la estadía en dichos conjuntos, algún problema en materia de relaciones intrafamiliares o a nivel de vecindario. Dichas deficiencias son lo suficientemente importantes para que los usuarios acepten las ampliaciones a pesar de la degradación que producen en el conjunto.

4. Finalmente, donde se observaron diferencias sustanciales respecto a las declaraciones de los vecinos de ambos condominios, fue cuando estos se refirieron a la localización del conjunto y a las características de la comuna de residencia. Llama la atención, por ejemplo, que el $90 \%$ de los entrevistados del conjunto Millaray II (La Pintana) comentaran el deseo de cambiarse de sector, manifestando un gran anhelo por residir en otra comuna. El $60 \%$ de estos ven a La Florida como la mejor opción, argumentando el deseo de vivir en otro tipo de lugar: con mejor equipamiento, áreas verdes y conectividad. La percepción de los habitantes de este conjunto respecto al sector en que viven es preocupante. El deseo de cambio se debe a los problemas comentados anteriormente respecto a la vida en copropiedad, como también por el entorno en que están inmersos. Los habitantes presentan un alto rechazo al sector en que viven, entre otros factores por la inseguridad que sienten, lo cual se ve intensificado dado a la calidad del equipamiento del entorno y a la localización del conjunto. En este condominio, el deseo es de irse "lo más lejos posible", surgiendo entrevistados que comentaron la esperanza de volver a sus ciudades de origen. Patricia Ramírez (49 años) se refirió al deseo de volver a Chillán para compartir con "otro tipo de gente, otro pueblo", refiriéndose con desprecio tanto a sus compañeros de block como a los de la comuna. Además de esto, se observó de manera constante el rechazo que los vecinos tienen del nivel de equipamiento o calidad del medio 85 
urbano en que están inmersos. Muchos de los vecinos reclamaban por la falta de basureros, expresaban molestia por el nivel de pavimentación, la gran cantidad de zonas "café" o sitios eriazos, la falta de vegetación, y el mal estado en que se encontraba, por ejemplo, la cancha de futbol contigua al conjunto. Comentaron lo desagradable que es el condominio, en especial en invierno, en que la formación de barro producto de la lluvia volvía aquellos espacios inutilizables. Tales situaciones "obligan", según ellos, a estar encerrados en el interior de sus viviendas. Ven estas como un refugio para evadir lo errático del espacio circundante.

Situación completamente distinta se observó en el conjunto Alberto Hurtado de la comuna de Lo Prado, en que si bien muchos de los habitantes manifiestan el deseo de cambio a una vivienda de propiedad individual (casas) comentaron también el deseo de permanecer en el sector en que actualmente residen. Para explicar lo anterior, es que los distintos entrevistados presentaron argumentos bastante similares. Prácticamente todos mencionaron que les gusta la comuna por los servicios que esta les entrega, comentando la presencia cercana de la Municipalidad, bancos, juzgados o lugares para el pago de cuentas (luz, agua o gas). Comentaron que es una comuna bastante comercial encontrándose "ferias, persas y almacenes por todos lados". Los entrevistados explicaron que no es necesario salir de la comuna porque en general tienen al alcance todo lo que necesitan. A lo anterior debe sumarse la buena conectividad que posee el conjunto, lo cual es valorado por los residentes, en especial la presencia de la estación de metro Lo Prado "a pocos minutos" de las puertas de sus casas. Respecto a los espacios públicos comunales, los vecinos poseen una buena percepción, comentando que tienen buenos parques y plazas. Explican que en términos generales hay varios espacios de recreación, para el juego de los niños, la realización de deportes o para el simple estar.

5. A modo de síntesis general es posible afirmar la existencia de ciertas tendencias respecto a la percepción que tienen los habitantes de sus conjuntos. Se observó entre otros aspectos, como el deseo de vivir en una vivienda en propiedad individual es un sentimiento común, producto principalmente de los problemas en materia de relaciones sociales entre los vecinos. Las responsabilidades compartidas, las características de los departamentos y el deficiente espacio público generan roces entre los residentes. Este último elemento es el que presenta menor aceptación por parte de los vecinos. En los espacios frente a las puertas de accesos se busca realizar una serie de actividades mientras que los espacios traseros dejaron de ser públicos hace muchos años luego de la apropiación de los residentes de los primeros pisos. La vivienda es el espacio mejor evaluado dado a que sirve como refugio y se constituye como el único lugar que no debe ser compartido. No obstante, los problemas espaciales y la calidad de las viviendas vienen a empañar este sentimiento de orgullo que muchos de los propietarios tienen de su inmueble.

\section{CONCLUSIONES}

Finalmente, y en relación al análisis de las entrevistas es posible plantear que la habitabilidad se está viendo profundamente transgredida en ciertos condominios sociales de nuestro país. Si bien no es factible extrapolar a nivel nacional o regional lo observado en estos dos casos de estudios, si es posible afirmar que un grupo importante de familias habitantes en la tipología de condominio elegida, presentan problemas graves de habitabilidad materializados en 
hacinamiento en las distintas dimensiones o escala de la vivienda. Las observaciones anteriores permiten afirmar:

1. La cantidad de viviendas en situación de hacinamiento es mayor que la informada por las fuentes oficiales. Si se entiende el hacinamiento como una situación en que producto de la falta de espacio las familias ven vulnerada, entre otros aspectos, la privacidad tanto entre los miembros de la familia como a nivel barrio, es que se puede afirmar que en Chile las familias en tal problemática superan al 17,16\% detectado por el MINVU (2004). A través de las entrevistas se observó que familias, por ejemplo, integradas por 6 individuos, en un departamento de 3 habitaciones y $40 \mathrm{~m}^{2}$ de superficie se siente en situación de hacinamiento, ya que el reducido metraje de la vivienda impide por ejemplo el estudio de los hijos, la lectura de un libro, el descanso personal o el desarrollo íntimo de parejas, entre muchas otras. El caso recién mencionado, según el sistema de medición usado en Chile no presenta hacinamiento, dado a que se observan menos de 2,5 personas habitando cada dormitorio. De esta forma, y entendiendo que son muchas más las combinaciones posibles de familias que no cumplen con el criterio de medición chileno y que no ven satisfechas sus necesidades producto de la falta de espacio, es que se puede decir que la primera hipótesis planteada se ha corroborado.

Efectivamente si se utilizara un método de medición como el usado en ciudades europeas ${ }^{3}$, el hacinamiento sería casi una condición innegable para muchas de las familias beneficiadas por los programas de gobierno implementados en las últimas décadas. En base a lo anterior, es que se plantea necesario idear un nuevo sistema de medición de esta patología, la cual permita incorporar la totalidad de familias en tal situación. Se propone la incorporación de una mirada cualitativa, basada en una concepción ampliada de lo que el hacinamiento produce.

2. El hacinamiento debe ser medido relacionando la escala de barrio y la localización de los conjuntos. Entendiendo las diversas escalas que posee la vivienda y que hay una serie de necesidades relacionadas con esta, que pueden o deben ser resueltas en aquellas dimensiones que van más allá de las 4 paredes que conformar la morada, es que se concluye que el hacinamiento, especialmente en condominios sociales, debería ser medido, estudiado e intervenido en relación a la escala de barrio y de la localización del condominio.

Los entrevistados fueron categóricos al afirmar que el espacio exterior es el lugar que presenta mayor rechazo por los habitantes. La densidad de estos conjuntos no guarda relación con la superficie de los espacios comunes. Estos lugares no permiten el adecuado desarrollo de los usuarios haciéndose muchas veces inviable la satisfacción de aquellas necesidades susceptibles de desarrollarse mediante "el uso de ciertos bienes compartidos" (Arcas et. al. 2011). En dicho espacio los vecinos pretenden realizar una serie de actividades que no es posible desarrollar al interior de las viviendas. Además, tales lugares son de responsabilidad compartida, siendo razón de discordias producto de la falta de compromisos que los usuarios poseen con él. Es así como las dimensiones, calidad y equipamiento del entorno, puede aumentar o disminuir la insatisfacción producida por el hacinamiento en que están inmersos.

\footnotetext{
${ }^{3}$ Autores como Blachere, plantean que para una familia de tres o más personas, menos de 18 $\mathrm{m}^{2}$ por habitante corresponde a hacinamiento, siendo necesario entre 14 y $22 \mathrm{~m}^{2}$ útiles para generar "confort modesto", y $18 \mathrm{~m}^{2}$ para otorgar un "confort medio" (Harrison, 1993: 162). En Suecia, por su parte, se consideran $20 \mathrm{~m}^{2}$ por persona para las familias modestas (Martínez, 2001). 
Respecto a lo anterior es que resulta interesante comparar la calidad de los condominios sociales analizados, con aquella solución habitacional ampliamente reproducida en el centro de Santiago formada por estudios o pequeños departamentos. En estos, a pesar de que muchas veces los estándares en términos de superficie son similares a los conjuntos estudiados, la sensación de hacinamiento no es un elemento común. Lo anterior podría fundamentarse dado a la cantidad y calidad del espacio comunitario que poseen los nuevos departamentos del centro, los cuales permiten la satisfacción de aquellas necesidades susceptibles de realizarse fuera de los límites convencionales de la vivienda. Problemas como el estacionamiento de los vehículos, el colgado de ropa, la realización de fiestas, etc., a pesar de que no pueden ser realizadas al interior de las 4 paredes de los departamentos no se constituyen como problemas dado a que se han previstos soluciones para evitar aquellos inconvenientes.

3. En relación a lo anterior, y al tercer objetivo específico planteado, es necesario agregar como conclusión que no sólo las características de la solución habitacional inciden en esta percepción de habitabilidad o de hacinamiento. De las entrevistas realizadas en la investigación, se desprendió como la variable localización del conjunto en la ciudad, puede determinar en gran medida la satisfacción de los residentes respecto a su hábitat. Sin duda, una de las observaciones más concluyentes tiene relación con como la localización de conjuntos de similares condiciones (año de llegada de los habitantes, cantidad de familias y departamentos, disposición de bloques de departamento) puede afectar de tal medida, que condominios como Millaray II de la comuna de La Pintana, se encuentran con un alto nivel de deterioro, en que la gran mayoría de los entrevistados manifiestan un profundo deseo por salir del conjunto que habitan. Se observa una falta de arraigo, pertenencia e identificación. Aquel concepto de territorialidad, que hace referencia a la utilización y defensa del territorio por parte de sus usuarios (Hall, 1989) no presenta ninguna luz de observarse. Tal situación resulta producto de una baja calidad del ambiente urbano, un sentimiento de inseguridad elevado y la localización periférica que hace que estos habitantes sientan un elevado grado de exclusión y de falta de oportunidades.

En estos casos, aquellas necesidades posibles de satisfacerse en la tercera escala de la vivienda (planteada por el INVI y que hace referencia a la vida de barrio) no cuentan con el soporte para poder llevarse a cabo. Actividades como el ocio, el paseo luego de una jornada laboral, el deporte al aire libre, entre otras, solo pueden realizarse si los habitantes de estos conjuntos recorren largos kilómetros hasta encontrar un parque o una cancha equipada que permita el desarrollo de tales actividades.

4. Dentro de las hipótesis del actual trabajo, es que se planteó que el hacinamiento no es solo consecuencia de un hábitat residencial ineficiente, sino que también se logra materializar como una causa y promotor de más hacinamiento. Tal hipótesis logró sustento con la actual investigación, ya que se ha observado que una de las principales razones por lo que las familias (al menos de los 2 conjuntos estudiados) realizan ampliaciones, es para paliar la falta de espacio al interior de las viviendas materializándose fundamentalmente en la construcción de nuevos dormitorios. Tal situación fue observada en las entrevistas realizadas, permitiendo plantear la existencia de un circulo vicioso en que las familias al intervenir sus viviendas realizan tal acción sobre los terrenos de dominio común, degradándolo hasta hacer que este pierda toda cualidad de espacio público. Al producirse tal situación, se incrementan las consecuencias que el hacinamiento produce, ya que se imposibilita el desarrollo de aquellas necesidades a desarrollar fuera de los límites que conforma la escala más íntima de la vivienda. 
http://revistas.uchile.cl/index.php/RU/index

\section{RECOMENDACIONES}

Finalmente es que surge de manera evidente proponer la construcción de viviendas de mayor tamaño y con mejores espacios públicos. Resulta interesante la existencia de actuales proyectos que consideran la opción de ampliación de las viviendas, situación que la tipología estudiada no presenta. Parece ser imprescindible también la exigencia de mejores diseño y equipamientos en el espacio circundante. Afortunadamente la tipología estudiada se ha dejado de construir, sin embargo en los nuevos conjuntos desarrollados se observan ciertos elementos que pueden reproducir los problemas anteriormente descritos. Es más, ciertas normativas siguen fomentando la generación de conjuntos propensos de generar hacinamiento. Por tal razón y buscando evitar la reproducción de los problemas observados anteriormente en los futuros conjuntos habitacionales es que propone:

1. Revisar la ordenanza del PRMS, el cual por ejemplo, dista diferencias a la hora de enunciar densidades en las comunas que no cuentan con un plan regulador como es el caso de Til- Til, Colina o Lampa. Habiéndose comentado las consecuencias de las altas densidades en este tipo de viviendas es que parece necesario la revisión del artículo 4.3 del Título $4^{\circ}$, el cual establece que para tales comunas la densidad bruta máxima para las áreas urbanizadas será de 150 $\mathrm{Hab} / \mathrm{Há}$, exceptuándose de esta norma "los proyectos de conjunto de viviendas sociales que se emplacen en predios de superficie no superior a 5 Há", los cuales pueden alcanzar una densidad máxima Bruta de hasta $300 \mathrm{Hab} / \mathrm{Há}$. Es decir, la normativa establece este tipo de diferencias que evidentemente se traducirán en diferentes condiciones de habitabilidad. Se propone para este caso, eliminar este tipo de diferencias a la hora de proyectar los conjuntos habitacionales.

2. En segundo lugar, y en relación a la escala de conjunto habitacional, se plantea la necesidad de revisar, por ejemplo, el Artículo 46 del Programa Fondo Solidario de Elección de Vivienda. Este regula el equipamiento que deben tener los nuevos conjuntos habitacionales acogidos a la Ley de Copropiedad Inmobiliaria (entre otros), estableciendo que para conjuntos que alberguen entre 30 a 70 viviendas, debe considerarse como mínimo la construcción de áreas verdes, plazas de juegos infantiles y área recreacional deportiva, siendo exigible la existencia de una sala multiuso solo en conjuntos que posean más de 70 viviendas. Considerando tanto lo recién mencionado como lo analizado anteriormente, es que cualquiera podría vaticinar que en los nuevos conjuntos habitacionales se observaran los problemas de convivencia producto de la falta de equipamiento para ciertas actividades. En los conjuntos de menos de 70 departamentos, seguirán sin contar con un espacio destinado para la realización de fiestas o celebraciones. A ninguno de los conjuntos futuros se les exigirá la existencia de una zona destinada como quincho, o para el colgado de ropa. Este último es de gran relevancia si se considera que la "tabla de espacio mínimos y usos mínimos para el mobiliario", exige para la aprobación de los nuevos proyectos tan solo una logia de $1,3 \mathrm{~m}^{2}$ por departamento. Con lo anterior se argumenta que los inconvenientes observados por la utilización del espacio público para la realización de este tipo de actividades seguirán siendo una constante. Modificaciones a tal artículo, se vuelven en extremo necesario si se pretende que en los futuros proyectos habitacionales no se repitan la gran cantidad de problemas expuestos anteriormente producto de la falta de equipamiento para la satisfacción de aquellas necesidades que no logran realizarse en el interior de la vivienda.

3. Respecto a lo anterior, y si bien se reconoce un avance a la hora de exigir la existencia de plazas y áreas deportiva, estás están reguladas por un Itemizado de carácter técnico que básicamente indica las normas que deben cumplir las diversas partidas para darle seguridad a 89 


\section{REVISTA DE}

\section{URBANISMO}

ISSN 0717-5051

http://revistas.uchile.cl/index.php/RU/index

las obras desarrolladas. Sería interesante que tales proyectos fueran regulados de manera más exhaustiva velando por la calidad de los espacios generados. Es necesario que la normativa reconozca la importancia de la calidad del espacio público y lo necesario de generar soluciones sustentables en el tiempo.

Respecto a estas zonas de uso público, es que se considera imprescindible el fortalecimiento de los espacios públicos a nivel comunal, la generación de imágenes de ciudad y la planificación estratégica de estas. Es evidente lo importante de generan proyectos de ciudades bajo criterios claros, consensuados y atingentes a las actuales posturas de diseño urbano. Así mismo es necesario lograr algún mecanismo que permita dar cumplimiento a lo enunciado por los instrumentos de planificación. No tiene mucho sentido proponer en planos áreas verdes y/o equipamiento si estas quedarán solo como proyectos. Las altas densidades que muchos de estos conjuntos poseen amerita que se lleven a cabo las áreas de recreación que los distintos instrumentos han establecido. Lo anterior haciendo referencia directa al conjunto Millaray II, el cual según el plan regulador comunal, debería estar a menos de 100 metros de un gran zona destinada como parque comunal. Este en la actualidad, se materializa en una gran "área café", sin equipamiento, arborización, ni nada que lo permita catalogar como un espacio público.

4. Finalmente, y considerando la importancia de la localización de los conjuntos es que se repite aquella recomendación que muchos teóricos han planteado, pero que dado el tema es necesario volver a comentar, y que tiene que ver con mejorar la localización de los futuros conjuntos a realizarse. Para lo anterior resulta necesario frenar el crecimiento de las ciudades, en particular de la región metropolitana, procurando de esta forma consolidar las zonas ya urbanizadas. 


\section{BIBLIOGRAFÍA}

ACEVES González, Francisco (Mayo- Agosto, 1997). La territorialidad punto nodal en la intersección, Revista Comunicación y Sociedad (30): 275-301.

ARCAS A., Joaquim, PAGÈS R., Anna, y CASALS T., Marina (Agosto 2011). El futuro del hábitat: repensando la habitabilidad desde la sostenibilidad. El caso español, Revista INVI 26(72): 6593.

ARAVENA, Susana, y SANDOVAL, Alejandra (2005). El diagnóstico de los pobladores "con techo". En: RODRÍGUEZ Alfredo y SUGRANYES Ana (Eds.). Los con techo, un desafío para la política de vivienda social. Santiago, Chile. Ediciones SUR. 123-137.

BORJA, Jordi, y MUXI Zaida (2000). El espacio público, ciudad y ciudadanía. Editorial Electa, Barcelona.

HALL T., Edward (1989). El lenguaje silencioso. Editorial Alianza. Madrid.

HARRISON Ogalde, Francisco (1993) Espacio construido y comportamiento. En: BRAVO Heitmann, Luis y MARTÍNEZ Corbella, Carlos (Eds.). Chile: 50 años de Vivienda Social 19431993. Valparaíso: Universidad de Val

GUTIÉRREZ García, Juan Pablo (2010). Blocks de vivienda social en Santiago: propuestas para mejorar su habitabilidad. Tesis (Arquitecto). Pontificia Universidad Católica de Chile, Facultad de Arquitectura, Santiago.

LENTINI, Mercedes, y PALERO, Delia (Agosto 1997). El hacinamiento: la dimensión no visible del déficit habitacional, Revista INVI 12(31): 23-32.

MARTÍNEZ Corbella, Carlos (2001). Algunas teorías sobré la vivienda de interés social: conceptos y características de un nuevo tipo de arquitectura. Valparaíso: Universidad de Valparaíso, Facultad de Arquitectura. 189p.

MINVU. Ministerio de Vivienda y Urbanismo (2004). El Déficit Habitacional en Chile: medición de los requerimientos de vivienda y su distribución espacial. Santiago, Ministerio de Vivienda y Urbanismo. 104p.

MINVU. Ministerio de Vivienda y Urbanismo (2004) (2). Chile un siglo de políticas en vivienda y barrio. Santiago. DITEC. Pehuén editores.

MINVU. Ministerio de Vivienda y Urbanismo (2011). Catastro 2011: Mapa Social de Campamentos. Resultados generales. [En línea] <http://www.minvu.cl/opensite_20110523144022.aspx > [consulta: 20 junio 2011]

MINVU. Ministerio de Vivienda y Urbanismo (2012). Condominios Sociales. [En línea] <http://www.minvu.cl/opensite_20070308155730.aspx> [consulta: 26 junio 2011]

POBLETE Toelg, Carolina (2001). Mejoramiento de los espacios comunitarios en condominios de vivienda social en la periferia de Santiago. Seminario de Vivienda Social (Arquitecto). Universidad de Chile, Facultad de Arquitectura y Urbanismo, Santiago. 152p. 
PUGA, Josefina (1983). Consecuencias sociales del déficit habitacional en los sectores urbanos de mínimo ingreso En: MAC DONALD, Joan (Editora). Vivienda social, reflexiones y experiencias. Santiago, Chile: Corporación de promoción universitaria. 199-215.

RODRÍGUEZ, Alfredo y SUGRANYES, Ana (2005). El problema de vivienda de los "con techo". En su: Los con techo, un desafío para la política de vivienda social. Santiago, Chile. Ediciones SUR. 59-78.

RUGIERO Pérez, Ana (Agosto 2000). Aspectos teóricos de la Vivienda en relación al Habitar, Revista INVI 15(40): 69-97.

TRIVELLI O., Pablo (Agosto 2009). Suelo urbano y equidad: Deuda pendiente con las ciudades. Revista Mensaje 58 (581): 350-353.

INVI UNIVERSIDAD DE CHILE. Instituto de la Vivienda (2005). Mejoramiento del parque habitacional. Universidad de Chile. Facultad de Arquitectura y Urbanismo, Santiago.

INVI UNIVERSIDAD DE CHILE. Instituto de la Vivienda (2004). Bienestar habitacional. Guía de diseño para un hábitat residencial sustentable. 